\title{
High-sensitivity terahertz imaging of traumatic brain injury in a rat model
}

\author{
Hengli Zhao \\ Yuye Wang \\ Linyu Chen \\ Jia Shi \\ Kang Ma \\ Longhuang Tang \\ Degang $\mathrm{Xu}$ \\ Jianquan Yao \\ Hua Feng \\ Tunan Chen
}




\title{
High-sensitivity terahertz imaging of traumatic brain injury in a rat model
}

\author{
Hengli Zhao, ${ }^{a, \dagger}$ Yuye Wang, ${ }^{a, b, c, \dagger}$ Linyu Chen,,${ }^{b, c}$ Jia Shi, ${ }^{b, c}$ Kang Ma, ${ }^{a}$ Longhuang Tang, ${ }^{b, c}$ Degang Xu, ${ }^{b, c, *}$ \\ Jianquan Yao,, ${ }^{\mathrm{b}, \mathrm{c}}$ Hua Feng, ${ }^{\mathrm{a}, \boldsymbol{}}$ and Tunan Chen ${ }^{\mathrm{a}, *}$ \\ ${ }^{a}$ Southwest Hospital, Third Military Medical University (Army Medical University), Department of Neurosurgery and Key Laboratory of Neurotrauma, \\ Chongqing, China \\ ${ }^{\mathrm{b} T i a n j i n ~ U n i v e r s i t y, ~ I n s t i t u t e ~ o f ~ L a s e r ~ a n d ~ O p t o e l e c t r o n i c s, ~ S c h o o l ~ o f ~ P r e c i s i o n ~ I n s t r u m e n t ~ a n d ~ O p t o e l e c t r o n i c s ~ E n g i n e e r i n g, ~ T i a n j i n, ~ C h i n a ~}$ \\ 'Tianjin University, Key Laboratory of Optoelectronics Information Technology (Ministry of Education), Tianjin, China
}

\begin{abstract}
We demonstrated that different degrees of experimental traumatic brain injury (TBI) can be differentiated clearly in fresh slices of rat brain tissues using transmission-type terahertz $(\mathrm{THz})$ imaging system. The high absorption region in $\mathrm{THz}$ images corresponded well with the injured area in visible images and magnetic resonance imaging results. The $\mathrm{THz}$ image and absorption characteristics of dehydrated paraffin-embedded brain slices and the hematoxylin and eosin (H\&E)-stained microscopic images were investigated to account for the intrinsic differences in the THz images for the brain tissues suffered from different degrees of TBI and normal tissue aside from water. The THz absorption coefficients of rat brain tissues showed an increase in the aggravation of brain damage, particularly in the high-frequency range, whereas the cell density decreased as the order of mild, moderate, and severe TBI tissues compared with the normal tissue. Our results indicated that the different degrees of TBI were distinguishable owing to the different water contents and probable hematoma components distribution rather than intrinsic cell intensity. These promising results suggest that $\mathrm{THz}$ imaging has great potential as an alternative method for the fast diagnosis of TBI. ๑ 2018 Society of PhotoOptical Instrumentation Engineers (SPIE) [DOI: 10.1117/1.JBO.23.3.036015]
\end{abstract}

Keywords: traumatic brain injury; terahertz imaging; terahertz spectroscopy.

Paper 170718RR received Nov. 6, 2017; accepted for publication Mar. 9, 2018; published online Mar. 28, 2018.

\section{Introduction}

Traumatic brain injury (TBI) is considered as a signature injury of modern warfare. Of the $\sim 2.5$ million service members who have deployed to Iraq and Afghanistan in support of Operations Iraqi Freedom and Enduring Freedom, more than 50,000 were wounded in action, and $30 \%$ to $50 \%$ of these injuries contained varying degrees of TBI. ${ }^{1,2}$ In addition, with fast development of the transportation and construction industries, TBI has become a leading cause of morbidity and disability in both industrialized and developing countries. ${ }^{3}$ After TBI occurs, cerebral contusion is usually accompanied by cerebral edema of varying extent, ${ }^{4}$ and the cerebral blood flow (CBF) within and around the contused tissues is lower than the ischemic-threshold, which leads to an ischemic state as "pericontusional penumbra." Edema and ischemia both contribute to the pathophysiological process of TBI. $^{6}$ In addition, the injury degree of brain function, the risk of complications, prognosis, and the selection of clinical treatment are all closely related to the degree of trauma. ${ }^{7}$ However, it is difficult to locate the cerebral functional areas and identify the necrotic brain tissues during traumatic brain surgery, which leads to extensive debridement, fatal complications, and permanent damage to brain function. Therefore, precise and accurate neurosurgery for TBI is very important in clinical practice. In addition to the experience of doctors, traditional medical imaging techniques such as computed tomography (CT) and

\footnotetext{
*Address all correspondence to: Degang Xu, E-mail: xudegang@ @ tju.edu.cn; Hua Feng, E-mail: fenghua8888@vip.163.com; Tunan Chen, E-mail: ctn@ tmmu.edu.cn

†These authors contributed equally.
}

magnetic resonance imaging (MRI) often provide useful information. However, these techniques also have many disadvantages, e.g., the use of a whole body imaging machine, high costs, system complexity, need for special coils, acquisition time, and patient claustrophobia. Therefore, more viable tools to help doctors better assess and operate after TBI are urgently wanted.

Terahertz-wave (THz-wave) lies between the infrared and microwave regions of the electromagnetic spectrum. Recently, $\mathrm{THz}$ imaging techniques have been studied as candidates for medical imaging as they provide a high sensitivity for the detection of biosamples with noninvasive and nonionizing characteristics. ${ }^{89}$ The application of $\mathrm{THz}$ imaging to cancer diagnosis has been widely investigated including brain, ${ }^{10,11}$ skin, ${ }^{12}$ breast, ${ }^{13}$ liver, ${ }^{14}$ colonic, ${ }^{15}$ and oral ${ }^{16}$ cancers. However, the potential of $\mathrm{THz}$ imaging applied for other nonneoplastic diseases remains largely unknown. As the fundamental principle behind biomedical diagnosis with $\mathrm{THz}$ waves depends on the distribution of biomolecules and water content, ${ }^{8}$ we proposed $\mathrm{THz}$ imaging may be able to reflect distinctive characteristics of TBI.

In this paper, we demonstrated that the TBI regions of fresh rat brain tissues with different degrees of TBI could be well distinguished by $\mathrm{THz}$ images, which has been compared with MRI results. After eliminating the influence of water, the $\mathrm{THz}$ image and absorption coefficients of paraffin-embedded samples have been measured, and the histological analysis with hematoxylin and eosin (H\&E) staining photography was performed. Our results indicated that the $\mathrm{THz}$ absorption coefficients of dehydrated rat TBI tissues and normal tissue increased with the

$1083-3668 / 2018 / \$ 25.00$ @ 2018 SPIE 
aggravation of brain damage, whereas the cell density decreased as the opposite trend. It is deduced that the identification of different degrees of TBI could be attributed to the difference of water content and probable hematoma components distribution.

\section{Experimental Methods}

Adult male Sprague-Dawley rats weighing 250 to $280 \mathrm{~g}$ were purchased from the Animal Center of the Third Military Medical University. All animal experiments were performed in accordance with the China Animal Welfare Legislation and were approved by the Third Military Medical University Committee on Ethics for the Care and Use of Laboratory Animals. The brain trauma model was established using a modified version of Feeney's method. ${ }^{17}$ After anesthetized with a pentobarbital $(50 \mathrm{mg} / \mathrm{kg}$ ) intraperitoneal injection, the head of the rat was mounted on a stereotaxic frame. The fur was shaved and routine disinfection was performed. The scalp was opened by a midline incision, and a right parietal craniotomy $(4.5 \mathrm{~mm}$ in diameter, $3-\mathrm{mm}$ posterior and $2.5-\mathrm{mm}$ lateral to the bregma) was performed with a dental drill, without laceration of the dura. A 30-g stainless steel rod with a small pillar $(4.5 \mathrm{~mm}$ in diameter, $5 \mathrm{~mm}$ in height) was dropped on the exposed dura from the height of 15,25 , and $35 \mathrm{~cm}$ to establish mild, moderate, and severe brain trauma, respectively. With the exception of the impingement, the surgical procedures performed on the trauma group were also performed on the sham operation group. Brain damage was visualized by staining brain slices with 2,3,5-triphenyltetrazolium chloride (TTC); ${ }^{18}$ neurologic impairment was evaluated by modified neurological severity score (mNSS); ${ }^{19}$ and brain water content (BWC) was measured using the dry-wet weight method, ${ }^{20}$ all performed as previously described to identify the different severities of TBI models (number $=6$ rats for each group). The comparison of different degrees of TBI was illustrated by T2-weighted MRI, specimen photo, and THz imaging. For the pathomorphological observation around TBI region, samples from each group were fixed in a formalin solution and dehydrated with paraffin to preserve the cell structure, then $3-\mu \mathrm{m}$-thick sections were sliced from the paraffin block and dyed with H\&E staining. ${ }^{20}$

The schematic diagram of the $\mathrm{THz}$ imaging system was shown in Fig. 1(a). An optically pumped $\mathrm{THz}$ gas laser (FIRL100, Edinburgh Instruments Ltd.) was used, which is capable of emitting high-power, tunable continuous $\mathrm{THz}$ wave. Such a $\mathrm{THz}$ source has the merits of good beam quality and higher signal-to-noise ratio owing to its higher energy output. The maximum $\mathrm{THz}$ output power was up to $150 \mathrm{~mW}$ at $2.52 \mathrm{THz}$. The Golay cells (GC-1P, Tydex Ltd.) were used as the detectors that can operate at room temperature. Considering the responsivity of the detectors, $\mathrm{THz}$ wave was modulated as sinusoidal signal using the chopper operating at $50 \mathrm{~Hz}$. The grounding and shielding of Golay cells were adopted to avoid electromagnetic interference and ensure their reliable performance. A wire-grid beam splitter separated the THz-wave into two beams, a signal beam for imaging and a reference beam, to reduce the image noises from slight power fluctuations. The signal beam was reflected by gold-coated flat mirrors and normally focused on the sample using an aspherical lens $(f=30 \mathrm{~mm})$. The sample was mounted on a computercontrolled $x-y$ linear motor stages that moved it through the focused beam in the horizontal plane. The terahertz beam transmitted through the sample was collimated with another aspherical lens and then was collected by a parabolic mirror

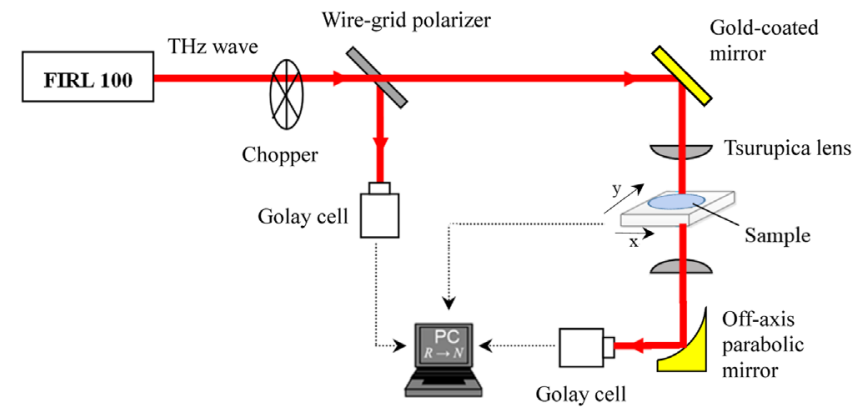

(a)

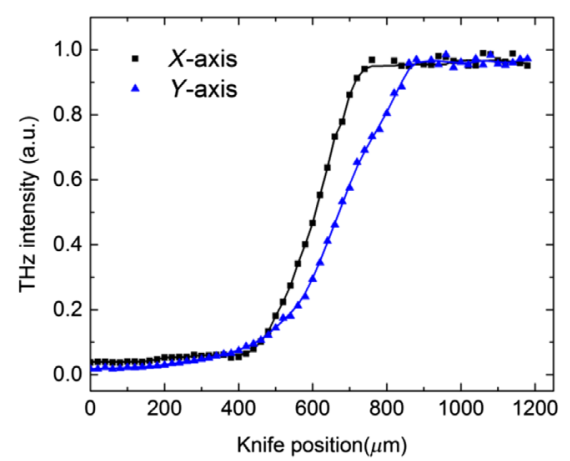

(b)

Fig. 1 (a) Schematic of terahertz transmission imaging setup and (b) the resolution of the imaging system measured by knife-edge method.

into a Golay cell detector. The fresh brain tissues with TBI were cut into $40-\mu \mathrm{m}$ thickness with a microtome (Leica, CM1950) and then sandwiched between two quartz slides (0.8-mm thick for each quartz slide). Especially, the oleic acid was used to cover the tissue for decreasing sample dehydration during the experiment. ${ }^{21,22}$ The spatial resolution of the $\mathrm{THz}$ imaging system was evaluated through detecting the $\mathrm{THz}$ focusing spot size at sample plane using the knife-edge method. Figure 1(b) show the measurement result at the scanning step of $20 \mu \mathrm{m}$. The $x$ - and $y$-diameters of the intensity distribution, measured as the $10 \%$ and $90 \%$ distance between the peak and bottom, were found to be 260 and $380 \mu \mathrm{m}$, respectively. The scanning step was set as $250 \mu \mathrm{m}$ in the experiment.

To investigate the absorption coefficients of TBI tissues in the $\mathrm{THz}$ range, a commercially available $\mathrm{THz}$ time-domain spectroscopy (TDS, Advantest Corp., TAS7500SP) in transmission geometry was used. The spectrum reached over 2.0 from $0.7 \mathrm{THz}$, with a dynamic range better than $70 \mathrm{~dB}$ under peak frequency around $0.7 \mathrm{THz}$ and the frequency resolution of 7.6 GHz. The paraffin-embedded tissues were cut into slices with the thickness of $1 \mathrm{~mm}$ (hundreds of times thicker than the classical paraffin section to increase $\mathrm{THz}$ absorption) for detection. Both sides of the samples were polished and kept parallel (with roughness $<50 \mu \mathrm{m}$ ). ${ }^{23}$ The diameter of $\mathrm{THz}$ pulse beam at the focus point was $<2.6 \mathrm{~mm}$ according to $2 r=1.22 \lambda / \mathrm{NA}$, where the numerical aperture NA is 0.167 . The measurements were performed at room temperature $\left(23^{\circ} \mathrm{C}\right)$ with dry air purge.

\section{Results and Discussions}

To validate the distinct alterations arising from different levels of trauma severity, we replicated previous studies reporting that manipulation of a modified version of Feeney's method led 


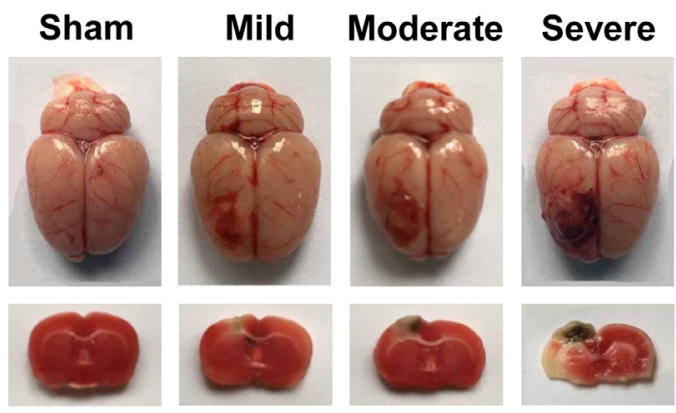

(a)

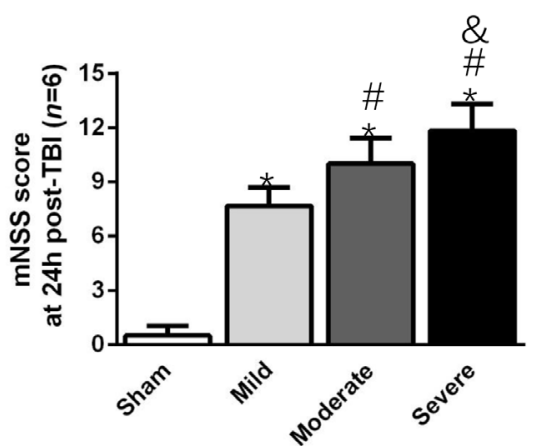

(b)

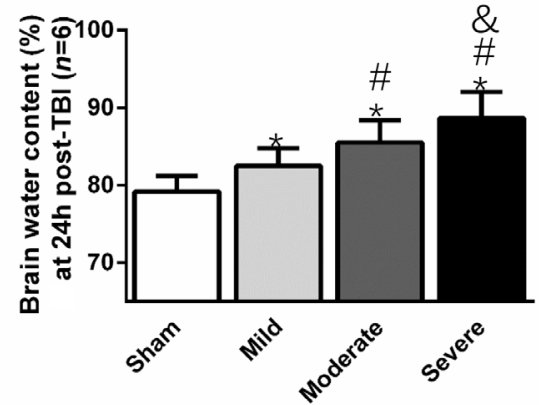

(c)

Fig. 2 (a) Histological manifestations of sham and different degrees of TBI tissues at gross and TTC staining examination; (b) mNSS and (c) BWC assessments of sham and different degrees of TBI groups.

to mild, moderate, and severe TBI, as shown in Fig. $2 .{ }^{17}$ Brain damage was revealed by a lack of TTC staining, which indicates that tissue is dehydrogenase deficient. Here, we demonstrated that, in contrast to no detectable histologic deficits in the sham group, the extent of brain damage corresponded well with TTC-stained brain damage volume in the following order of severity: severe $>$ moderate $>$ mild as the representative brain slices exhibited, as shown in Fig. 2(a). Moreover, to test whether the consequent behavioral abnormalities reflected the three different TBI severity levels, the animals underwent $\mathrm{mNSS}$ at $24 \mathrm{~h}$ post-TBI, just prior to the histological examination as discussed above. In complete agreement with the TBI severity of histological damage, all three behavioral outcome measures revealed that such neurological deficits were in parallel with the degree of TTC brain damage volume $\left(^{*} p<0.05\right.$ versus sham; ${ }^{\#} p<0.05$ versus mild injury; ${ }^{\&} p<0.05$ versus moderate injury), whereas animals in sham group significantly performed better than any of the TBI-exposed animals, as shown in Fig. 2(b). Due to the fact that cerebral edema is an important indicator to reflect the severity of TBI, we then measured BWC in different groups at $24 \mathrm{~h}$ post-TBI by dry-wet weight method. In agreement with the severity of TBI-induced histologic and behavioral deficits, brain edema also distinctively exhibited three levels of injury corresponding to the mild, moderate, and severe $\left({ }^{*} p<0.05\right.$ versus sham; ${ }^{\#} p<0.05$ versus mild injury; ${ }^{\&} p<0.05$ versus moderate injury), shown in Fig. 2(c).

Visual, THz, and MR images for fresh brain tissues without and with different degrees of TBI were shown in Fig. 3. We used T2-weighted MR imaging to observe the condition of TBI in vivo. Figure 3(a) shows the brightness of the MR images in the TBI regions with high signal was higher than that for the normal regions, indicating that the water content in the brain tissue with TBI was higher than that in the normal tissue. ${ }^{24}$
Moreover, different degrees of high signal appeared in the injured area were in good agreement with the order of damage severity: severe $>$ moderate $>$ mild. The high signal was not found in the sham group. After the MR scanning, the fresh specimens obtained were pruned at $-2^{\circ} \mathrm{C}$ using a microtome until the coronal plane match with MR images and cut into uniform slices for the THz imaging measurement, as shown in Fig. 3(b). Figure 3(c) shows the $\mathrm{THz}$ images of fresh brain tissues with different degrees of TBI and the sham group based on $\mathrm{THz}$ transmission imaging system. Each pixel was described using

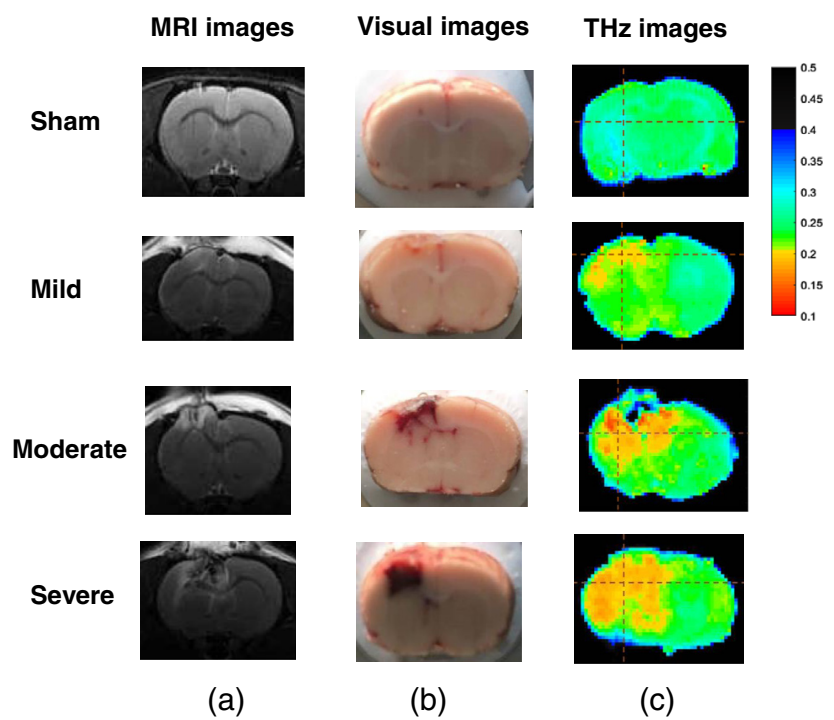

Fig. 3 (a) MR, (b) visual, and (c) THz images of fresh brain tissues without and with different degrees of TBI. 
the intensity transmittance of the $\mathrm{THz}$ wave, where a lower $\mathrm{THz}$ transmittance means the larger sample absorption. It is clearly seen that the $\mathrm{THz}$ transmittance image in the sham group looks uniform, with the average transmittance of $23 \%$. The $\mathrm{THz}$ images of different degrees of TBI tissues depicted obvious difference compared with sham group. The average transmittance values of the abnormal regions for mild, moderate, and severe TBI tissues were about $20 \%, 19 \%$, and $19 \%$, respectively, which were lower than those for the normal brain tissue. The volume of abnormal regions gradually increased for mild, moderate, and severe TBI tissues and the location of the TBI regions shown in the $\mathrm{THz}$ images was similar to those of the corresponding MR image. As the water content in brain tissue increases in parallel with the severity of TBI, the obtained MR and THz images indicated that water can be used as the primary agent in $\mathrm{THz}$ TBI imaging, as is the case for $\mathrm{THz}$ brain tumor imaging. ${ }^{25}$ Moreover, our results also found the minor $\mathrm{THz}$ image contrast differences in sham group were corresponded with the gray and white matter areas, as shown in visual image. The transmittance of $\mathrm{THz}$ wave was a little higher for the regions where the tissue color in visual image was white. This is due to the myelin in the white matter is mainly composed of lipids, whose absorbance of $\mathrm{THz}$ wave is lower than that of water, as in our previous study described. ${ }^{26}$ The distribution of white matter in the $\mathrm{THz}$ image was similar to those in the corresponding T2-weighted MR image.

Figure 4 shows the detailed profile of the transmittance values along the horizontal and vertical lines of Fig. 3(c). For the TBI tissue, the transmittance of the TBI region was lower than $21 \%$, whereas the transmittance for the normal brain tissues was rarely lower than $21 \%$. Then, the threshold value of $21 \%$ was chosen to differentiate TBI region from normal tissue in Fig. 3(c). The margins of the traumatic injury can be distinguished from the normal regions in the $\mathrm{THz}$ images. It is noted that the size of TBI regions indicated by $\mathrm{THz}$ image was larger than those detected using visual and MR image. Given the spatial resolution of $140 \times 140 \mu \mathrm{m}^{2}$ for MRI image, the relative low spatial resolution for $\mathrm{THz}$ image is one possible reason. This suggests the high-resolution $\mathrm{THz}$ imaging methods are necessary in future to accurately assess the TBI range. On the other hand, as the cellular hydration state and activity can greatly affect the information of $\mathrm{THz}$ wave, ${ }^{9}$ we postulated
$\mathrm{THz}$ imaging could indicate the presence of "pericontusional penumbra" around the necrotic tissues under the conditions of edema and ischemia. Considering the result well agrees with the MRI and has a high sensitivity, THz imaging technique could be a useful tool to determine the severity of TBI and complement conventional radiological techniques.

Brain edema is a pathological phenomenon of increased brain volume resulting from increased water in the brain, which can be divided into cytotoxic brain edema (intracellular), vasogenic brain edema, interstitial brain edema, and osmotic brain edema subtypes. Though the precise molecular mechanisms acting following TBI remain to be elucidated, brain edema is an important target and prognostic indicator in the treatment of TBI. ${ }^{27,28}$ Aside from water, some studies have reported that $\mathrm{THz}$ biomedical diagnostic imaging can be achieved using either the physical properties of cells or tissues, such as structure or density, or the chemical properties, such as protein or ion concentrations. ${ }^{25,29,30}$ To investigate whether intrinsic properties contribute to the ability of $\mathrm{THz}$ to distinguish different degrees of TBI, fresh brain tissues were dehydrated by replacing the water with paraffin for $\mathrm{THz}$ imaging and TDS detections, and then $H \& E$ staining was used to examine the differences among different degrees of TBI at the cellular level.

Figure 5 shows the $\mathrm{THz}$ image of $300-\mu \mathrm{m}$-thick paraffinembedded brain tissues without and with different degrees of TBI. It is seen that the location and size of the TBI region shown in the $\mathrm{THz}$ images were similar to those of the visual images. The average $\mathrm{THz}$ transmittance values of the abnormal regions for mild, moderate, and severe TBI tissues were about $43 \%, 32 \%$, and $28 \%$ respectively, which were lower than $47 \%$ for the normal brain tissue. This supports that the $\mathrm{THz}$ images of different degrees of TBI tissues are affected by differences in other biological factors besides the water content.

Furthermore, the THz absorption coefficients of dehydrated paraffin samples from sham and different degrees of TBI tissues were shown in Fig. 6. Six sample pieces for each group were used for the detection. Considering the most serious area of brain edema and the most obvious area of histopathological changes are located in the pericontusional region, the timedomain $\mathrm{THz}$ pulses around the TBI region were collected at three measurement points for each sample, denoted by red dashed circle in Fig. 5. Every measurement point was repeatedly

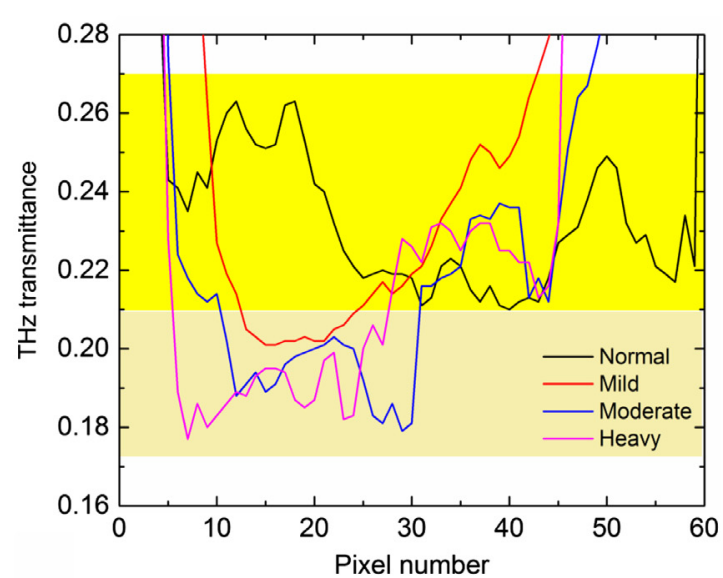

(a)

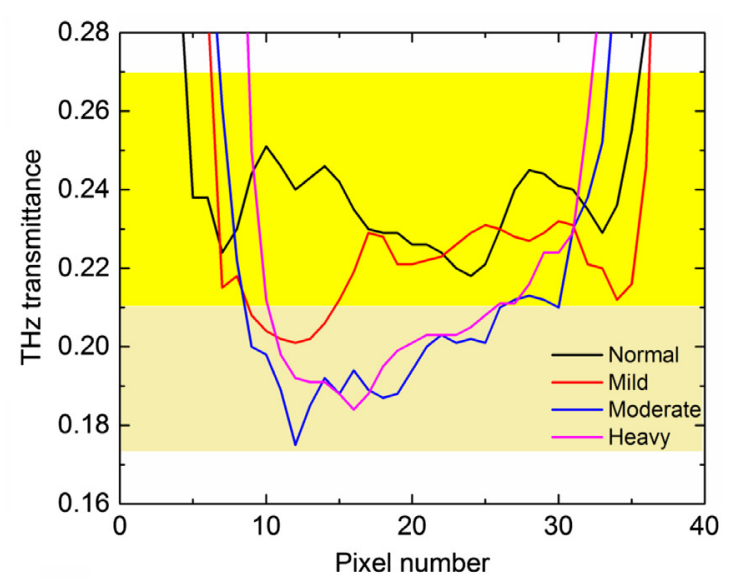

(b)

Fig. $4 \mathrm{THz}$ transmittance on the (a) horizontal and (b) vertical lines of Fig. 3(c). 


\section{Sham}
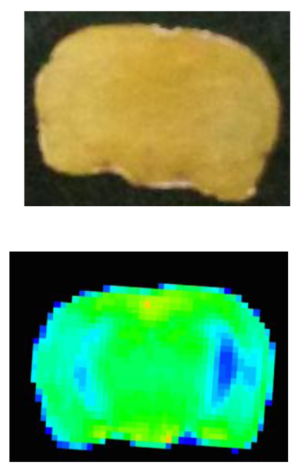

Mild
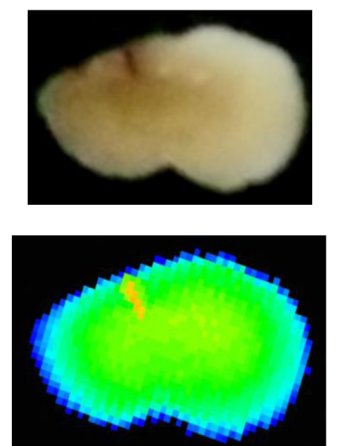

Moderate
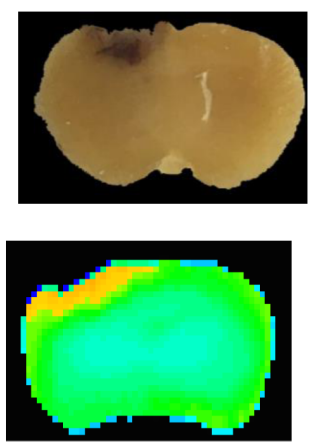

Severe

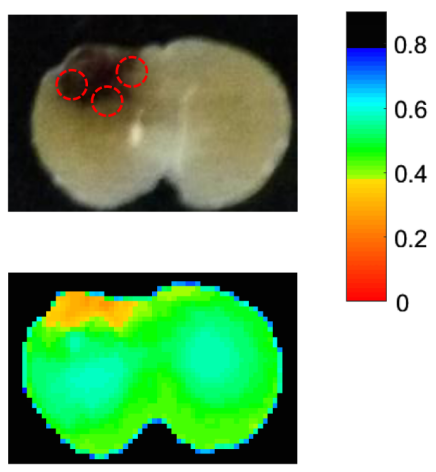

Fig. 5 Visual and THz images of paraffin-embedded brain tissues without and with different degrees of TBI.

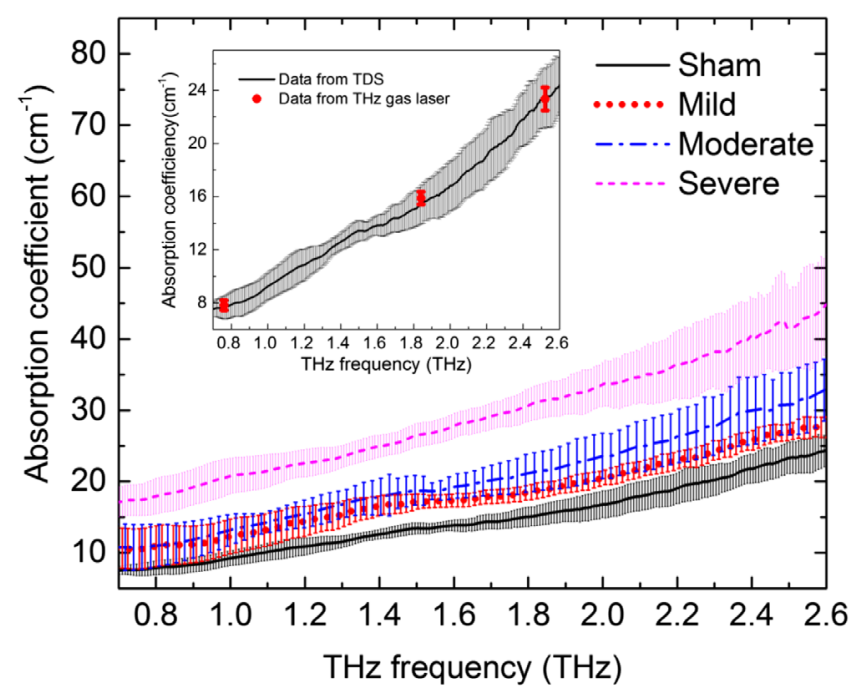

Fig. 6 Absorption coefficient of the paraffin-embedded brain samples from sham and different degrees of TBI tissues. tested 6 times with the setting of 1024 times scan for each measurement. The statistical mean value was used for the analysis. Within the measurement range of 0.7 to $2.6 \mathrm{THz}$, the averaged absorption coefficients monotonously increased with the THz frequency, as shown in Fig. 6. The error bars showing the standard deviation of the averaged values represent the variation over different chosen points for one kind of group. For the comparison with the $\mathrm{THz}$ imaging system mentioned above, absorption coefficients at different frequencies for sham group were also measured with $\mathrm{THz}$ gas laser, as presented in the inset. The inset illustrates that there was a better link between these measurements with the two systems. On the whole, the absorption coefficients of the dehydrated samples were $<50 \mathrm{~cm}^{-1}$ below $2.52 \mathrm{THz}$, which are much lower than that of pure water. The absorption difference of THz TBI imaging was dominated by the water content in all. By comparing the terahertz absorption coefficient spectra, it should be highlighted that the average values of $\mathrm{THz}$ spectra for TBI tissues were larger than those for normal tissue. The error bars for moderate and mild brain injured tissues overlapped, thus, mild and
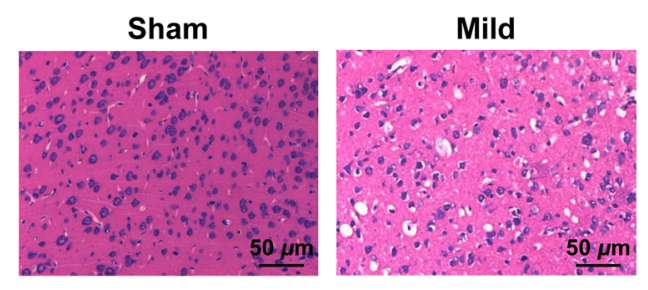

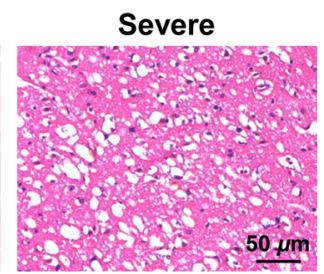

(a)

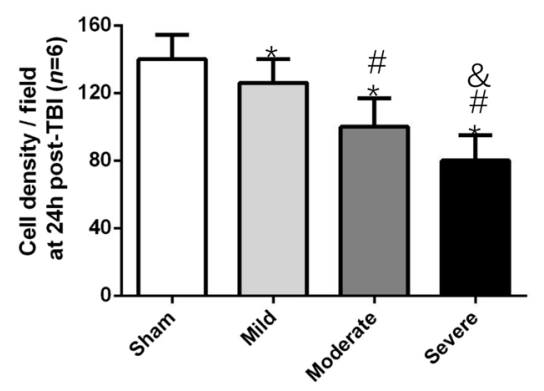

(b)

Fig. 7 (a) H\&E-stained microscope images of sham and different degrees of TBI tissues and (b) cell density of sham and different degrees of TBI tissues. 
moderate damages cannot be clearly distinguished within the error of the measurement. Overall, the averaged absorption of the $\mathrm{THz}$ wave increased as the order of normal tissue, mild, moderate, and severe TBI tissues, particularly in the highfrequency range, which agreed well with the order of brain damage severity. It is reasonable to speculate that biological factors besides the water content also account for the differences in the terahertz absorption spectra among different degrees of TBI tissues and normal tissue.

H\&E staining of brain tissue slice in sham group showed the nerve cell structure was clear and complete tightly packed. However, TBI led to the cell structure organized loosely and accompanied by cellular density decreased with cytoplasmic vacuolization in the following order of severity: mild > moderate $>$ severe, as shown in Fig. 7(a). In other words, the more severe the TBI was, the lower the cell density was $\left({ }^{*} p<0.05\right.$ versus sham; ${ }^{\#} p<0.05$ versus mild injury; ${ }^{\&} p<0.05$ versus moderate injury), indicated by Fig. 7(b). It could be suggested that cell density has minor effect on the $\mathrm{THz}$ spectral differences regarding distinguishing the severity of TBI when the effect of water was eliminated. Previous studies have demonstrated that the absorption of blood and iron contributed to the biomedical diagnostics of THz spectroscopy. ${ }^{31,32}$ Because more red blood cells, as well as their decomposed hemoglobin and iron deposition, appear in the pericontusional areas after severe TBI, these are the possible origins of the absorption spectral difference.

\section{Conclusion}

We obtained $\mathrm{THz}$ transmission images of fresh brain tissues without and with different degrees of TBI from a rat model. The TBI regions of fresh rat brain tissues with different degrees of TBI can be well distinguished by $\mathrm{THz}$ images, which have been compared with MRI results. After eliminating the influence of water, $\mathrm{THz}$ image and the absorption coefficients of dehydrated paraffin samples from sham and different degrees of TBI tissues were also measured by the imaging system and a $\mathrm{THz}$ time-domain spectroscopy, respectively. H\&E staining was used to examine the differences among different degrees of TBI at the cellular level. The experimental results show that the $\mathrm{THz}$ absorption coefficients of dehydrated rat normal tissue and TBI tissues increased with the aggravation of brain damage, whereas the cell density decreased as the order of mild, moderate, and severe TBI tissues compared with the normal tissue. Therefore, THz-wave has good capability to distinguish different degrees of TBI based on its remarkable sensitivity to track water content and distribution changes other than the effect of intrinsic cell density. Moreover, we hypothesized that the hematoma components after TBI may be the origin of differences in the terahertz absorption spectra among different degrees of TBI tissues and normal tissue aside from water, which need further exploration. It is anticipated that $\mathrm{THz}$ imaging technique could be employed as a complementary label-free technique for surgeons to quickly determine the location and size of TBI region, as well as the "pericontusional penumbra" around the necrotic tissues.

\section{Disclosures}

The authors have no relevant financial interests in this article and no potential conflicts of interest to disclose.

\section{Acknowledgments}

This work was supported by the National Basic Research Program of China (973) (2015CB755400), the National Key Research and Development Projects (2016YFC0101000), the National Natural Science Foundation of China (61775160), China Postdoctoral Science Foundation (2016M602954), Postdoctoral Science Foundation of Chongqing (Xm2016021), and Joint Incubation Project of Southwest Hospital (SWH2016LHJC04, SWH2016LHJC01).

\section{References}

1. V. L. Kristman et al., "Methodological issues and research recommendations for prognosis after mild traumatic brain injury: results of the International Collaboration on Mild Traumatic Brain Injury Prognosis," Arch. Phys. Med. Rehabil. 95(3), S265-S277 (2014).

2. D. G. Kang, R. A. Lehman, Jr., and E. J. Carragee, "Wartime spine injuries: understanding the improvised explosive device and biophysics of blast trauma," Spine J. 12(9), 849-857 (2012).

3. P. Reilly, "The impact of neurotrauma on society: an international perspective," Prog. Brain Res. 161, 3-9 (2007).

4. E. A. Winkler et al., "Cerebral edema in traumatic brain injury: pathophysiology and prospective therapeutic targets," Neurosurg. Clin. N. Am. 27(4), 473-488 (2016).

5. H. M. Wu et al., "Redefining the pericontusional penumbra following traumatic brain injury: evidence of deteriorating metabolic derangements based on positron emission tomography," J. Neurotrauma 30(5), 352-360 (2013).

6. M. W. Greve and B. J. Zink, "Pathophysiology of traumatic brain injury," Mt. Sinai J. Med. 76(2), 97-104 (2009).

7. D. K. Sandsmark, "Clinical outcomes after traumatic brain injury," Curr. Neurol. Neurosci. Rep. 16(6), 52 (2016).

8. N. Bajwa et al., "Non-invasive terahertz imaging of tissue water content for flap viability assessment," Biomed. Opt. Express 8(1), 460-474 (2017).

9. J. Shi et al., "Biomedical diagnosis of cerebral ischemia with continuous-wave THz imaging," in 41st Int. Conf. on Infrared, Millimeter, and Terahertz Waves (IRMMW-THz) (2016).

10. S. Yamaguchi et al., "Brain tumor imaging of rat fresh tissue using terahertz spectroscopy," Sci. Rep. 6, 30124 (2016).

11. Y. B. Ji et al., "Terahertz reflectometry imaging for low and high grade gliomas," Sci. Rep. 6, 36040 (2016).

12. C. S. Joseph et al., "Imaging of ex vivo nonmelanoma skin cancers in the optical and terahertz spectral regions optical and terahertz skin cancers imaging," J. Biophotonics 7(5), 295-303 (2014).

13. M. R. Grootendorst et al., "Use of a handheld terahertz pulsed imaging device to differentiate benign and malignant breast tissue," Biomed. Opt. Express 8(6), 2932-2945 (2017).

14. L. Rong et al., "Terahertz in-line digital holography of human hepatocellular carcinoma tissue," Sci. Rep. 5, 8445 (2015).

15. C. B. Reid et al., "Terahertz pulsed imaging of freshly excised human colonic tissues," Phys. Med. Biol. 56(14), 4333-4353 (2011).

16. Y. C. Sim et al., "Terahertz imaging of excised oral cancer at frozen temperature," Biomed. Opt. Express 4(8), 1413-1421 (2013).

17. D. M. Feeney et al., "Responses to cortical injury: I. Methodology and local effects of contusions in the rat," Brain Res. 211(1), 67-77 (1981).

18. S. Yu et al., "Severity of controlled cortical impact traumatic brain injury in rats and mice dictates degree of behavioral deficits," Brain Res. 1287, 157-163 (2009).

19. M. Lu et al., "Global test statistics for treatment effect of stroke and traumatic brain injury in rats with administration of bone marrow stromal cells," J. Neurosci. Methods 128(1-2), 183-190 (2003).

20. C. Zhang et al., "Expression of aquaporin-4 and pathological characteristics of brain injury in a rat model of traumatic brain injury," Mol. Med. Rep. 12(5), 7351-7357 (2015).

21. Y. Wang et al., "Study of water concentration measurement in thin tissues with terahertz-wave parametric source," Opt. Express 18(15), 15504-15512 (2010).

22. Y. Y. Wang et al., "Terahertz-wave water concentration and distribution measurement in thin biotissue based on a novel sample preparation," Phys. Med. Biol. 56(14), 4517-4527 (2011). 
23. K. Meng et al., "Terahertz pulsed spectroscopy of paraffin-embedded brain glioma," J. Biomed. Opt. 19(7), 077001 (2014).

24. F. Brabazon et al., "[18F]FDG-PET combined with MRI elucidates the pathophysiology of traumatic brain injury in rats," J. Neurotrauma 34(5), 1074-1085 (2017).

25. S. J. Oh et al., "Study of freshly excised brain tissues using terahertz imaging," Biomed. Opt. Express 5(8), 2837-2842 (2014).

26. Y. Zou et al., "Terahertz spectroscopic diagnosis of myelin deficit brain in mice and rhesus monkey with chemometric techniques," Sci. Rep. 7, 5176 (2017).

27. C. Werner and K. Engelhard, "Pathophysiology of traumatic brain injury," Br. J. Anaesth. 99(1), 4-9 (2007).

28. J. H. Ryu et al., "Induced and sustained hypernatremia for the prevention and treatment of cerebral edema following brain injury," Neurocrit. Care 19(2), 222-231 (2013).

29. L. Shi et al., "Terahertz spectroscopy of brain tissue from a mouse model of Alzheimer's disease," J. Biomed. Opt. 21(1), 015014 (2016).

30. J. B. Masson et al., "Ionic contrast terahertz near-field imaging of axonal water fluxes," Proc. Natl. Acad. Sci. U. S. A. 103(13), 4808-4812 (2006).

31. C. B. Reid et al., "Terahertz time-domain spectroscopy of human blood," IEEE J. Biomed. Health Inf. 17(4), 774-778 (2013).

32. M. T. Ruggiero et al., "The role of terahertz polariton absorption in the characterization of crystalline iron sulfate hydrates," Phys. Chem. Chem. Phys. 17(14), 9326-9334 (2015).

Hengli Zhao received his BS degree in medicine in 2013 from West China Medical Center, Sichuan University, China. He is a doctoral student in the Department of Neurosurgery, Southwest Hospital, Third Military Medical University (Army Medical University), China. His research interests are cerebrovascular disease and brain trauma.

Yuye Wang received her $\mathrm{PhD}$ in physics electronics from Tianjin University, Tianjin, China, in 2009. From 2015 to present, she is a postdoctoral fellow at the Department of Neurosurgery, Southwest Hospital, Third Military Medical University. And she is an associate professor at Tianjin University from 2011 to present. Her current research interests include high-power terahertz-wave source and terahertz-wave imaging application.

Linyu Chen is a master's student at School of Precision Instrument and Opto-Electronics Engineering, Tianjin University, Tianjin, China. His research interest is terahertz imaging.

Jia Shi received his BS degree in electronic information science and technology from Tianjin Polytechnic University, Tianjin, China, in 2013. Currently, he is working toward his $\mathrm{PhD}$ at the School of
Precision Instrument and Opto-Electronics Engineering, Tianjin University, Tianjin, China. His research interests include optical fiber sensors and terahertz imaging.

Kang Ma is a master's student at the Department of Neurosurgery, Southwest Hospital, Third Military Medical University (Army Medical University), China. His research interests are cerebrovascular disease and brain trauma.

Longhuang Tang received his bachelor's degree from the School of Optoelectronic Information, University of Electronic Science and Technology of China, Chengdu, China, in 2015. Since 2015, he has been working toward his PhD, majoring in optoelectronics and photonics technology, at the Institute of Laser and Opto-Electronic, Tianjin University, Tianjin, China. His research interests include terahertz sources based on nonlinear optics and terahertz applications in biomedical fields.

Degang $\mathrm{Xu}$ received his $\mathrm{PhD}$ in physical electronics from Tianjin University, Tianjin, China, in 2005. Currently, he is a full professor at Tianjin University. He has published more than 40 refereed journal articles in optoelectronics, nonlinear optics, and high-power laser. His current research interests include terahertz generation, amplification and detection, and their applications.

Jianquan Yao is currently a full professor and the director of Institute of Laser and Opto-Electronics in Tianjin University. He has long been engaged in the research of all-solid laser, nonlinear optics frequency conversion, and terahertz science and technology. His theory of precise calculation of optimum phase matching of biaxial crystal has been called as the "Yao and Fahlen Technology." In 1997, he was elected as the academician of Chinese Academy of Science.

Hua Feng is currently the director and a full professor at the Department of Neurosurgery. During his four-decade career in neurosurgery, he has investigated military application and research of brain science, interdisciplinary research and transformation of physics and biomedicine, treatment for nervous system trauma, and reconstruction of neurological function. He holds 36 patents. He has published 23 book chapters and is the author and coauthor of more than hundreds of peer-reviewed papers.

Tunan Chen received his PhD in neurosurgery from the Third Military Medical University, Chongqing, China, in 2012. Currently, he is a lecturer at the Department of Neurosurgery, Southwest Hospital, Third Military Medical University. His research interests include terahertz biomedical application, interdisciplinary research, and clinical transformation of physical and biomedical. 